\title{
Malignant Hypertension and Hypertensive Encephalopathy in Primary Aldosteronism Caused by Adrenal Adenoma
}

\author{
Luiz Aparecido Bortolotto, Fernando Henpin Yue Cesena, Fabio Biscegli Jatene, Hélio Bernardes Silva
}

São Paulo, SP - Brazil

Two cases are reported as follows: 1) 1 female patient with accelerated-malignant hypertension secondary to an aldosterone-producing adrenal adenoma; and 2) 1 female patient with adrenal adenoma, severe hypertension, and hypertensive encephalopathy. This association is a rare clinical finding, and malignant hypertension may modify the hormonal characteristic of primary aldosteronism, making its diagnosis more difficult. The diagnosis of primary aldosteronism should be considered in patients with malignant hypertension or hypertensive encephalopathy if persistent hypokalemia occurs. Identification of primary aldosteronism is of paramount importance for the patient's evolution, because the surgical treatment makes the prognosis more favorable.

Primary aldosteronism (Conn's syndrome) due to adrenal adenoma is a very rare and potentially curable cause of arterial hypertension ${ }^{1,2}$. Contrary to what Conn et al reported in 1964, we currently know that hypertension caused by adrenal adenoma may behave in a malignant way ${ }^{1,2}$.

We report the cases of 2 female patients with severe arterial hypertension, 1 with accelerated malignant hypertension and the other with hypertensive encephalopathy secondary to primary aldosteronism due to an aldosteroneproducing adrenal adenoma. The major characteristics of primary aldosteronism and the particularities of its association with accelerated malignant hypertension are reviewed. In addition, the important role played by the renin-angiotensin-aldosterone system in the development of accelerated malignant hypertension is discussed, as is its behavior when associated with primary aldosteronism.

Instituto do Coração do Hospital das Clínicas - FMUSP

Mailing address: Luiz Aparecido Bortolotto - InCor - Unidade de Hipertensão Av. Dr. Enéas C. Aguiar, 44 - 05403-000 - São Paulo, SP, Brazil - E-mail: hipluiz@incor.usp.br

English version by Stela Maris C. e Gandour
Case 1 -The patient is a 48-year-old female admitted to the Hypertension Unit at InCor with a history of arterial hypertension since her second pregnancy 20 years before. On ambulatory follow-up, the patient's condition worsened despite the therapeutic scheme with 4 drugs. The patient reported 3 episodes of hypertensive crisis a few months before hospital admission, and those episodes were characterized by blood pressure levels of $230 / 130 \mathrm{mmHg}$, important headache, nauseas, muscular weakness, and cramps. Hypokalemia $(\mathrm{K}=2.6 \mathrm{mEq} / \mathrm{L})$ was then evidenced. The patient was taking losartan $(100 \mathrm{mg} /$ day $)$ and clonidine $(0.20 \mathrm{mg} /$ day). On hospital admission, the physical examination revealed a patient in good general condition, with a heart rate of $70 \mathrm{bpm}$, and blood pressure of $220 / 140 \mathrm{mmHg}$. The patient showed no abnormality on the cardiac, pulmonary, and abdominal examinations. Funduscopy showed hemorrhages and cotton-wool exudates in the right eye. The complementary laboratory tests showed normal renal function (serum urea of $19 \mathrm{mg} / \mathrm{dL}$ and serum creatinine of $0.7 \mathrm{mg} / \mathrm{dL}$ ) and normal urinary sediment. The abdominal ultrasonography showed kidneys of normal size and morphology, and the renal arteriography showed no stenosis of the renal artery. The measurements of vanillylmandelic acid and metanephrines in the 24-hour urine were normal. In the initial measurement of plasma electrolytes, $\mathrm{K}$ was $2.9 \mathrm{mEq} / \mathrm{L}$ and $\mathrm{Na}$ 12 control during hospitalization was initially unsatisfactory, the introduction of spironolactone ( $300 \mathrm{mg} /$ day) being required. The drug was maintained for 10 days, and normalization of serum potassium and improvement in blood pressure control were obtained. The patient underwent right adrenalectomy, and a $2.0-\mathrm{cm}$ yellow nodule was found in the cortical zone. The anatomicopathological examination diagnosed cortical adrenal adenoma (figs. 1 and 2). The patient evolved uneventfully in the postoperative period and was discharged from the hospital asymptomatic. She was then taking clonidine $(0.4 \mathrm{mg} / \mathrm{day})$ and maintained her blood pressure at $130 / 90 \mathrm{mmHg}$ and her serum potassium levels within the normal range. Three months after surgery, she maintained her blood pressure levels under control taking 2 drugs $(0.4 \mathrm{mg} /$ day of clonidine and $5 \mathrm{mg} /$ day of amlodipine); 
her serum potassium level was then $4.9 \mathrm{mEq} / \mathrm{L}$, her plasma renin activity was $0.8 \mathrm{ng} / \mathrm{mL} / \mathrm{h}$, and her plasma aldosterone level was $8.4 \mathrm{ng} / 100 \mathrm{~mL}$. On that occasion, funduscopy revealed the absence of exudates and hemorrhages. One year after adrenalectomy, the patient remains without hypertensive crises; her blood pressure is $140 / 90 \mathrm{~mm} \mathrm{Hg}$, and she is taking fewer medications than she did before the surgery $(0.4 \mathrm{mg} /$ day of clonidine, $50 \mathrm{mg} /$ day of atenolol, and $10 \mathrm{mg} /$ day of amlodipine). Her serum potassium is $5.0 \mathrm{mEq} / \mathrm{L}$, her plasma renin activity is $1.8 \mathrm{ng} / \mathrm{mL} / \mathrm{h}$, and her plasma aldosterone level is $13.6 \mathrm{ng} / 100 \mathrm{~mL}$.

Case 2 - A 34-year-old female patient was admitted to the Hypertension Unit at InCor complaining of headache and blurred vision. She reported having systemic arterial hypertension, which had been diagnosed during pregnancy 9 years before and was difficult to control in the last 4 years. During this period, she required several hospitalizations because of hypertensive crises. The patient was taking $50 \mathrm{mg} /$ day of hydrochlorothiazide, $60 \mathrm{mg} /$ day of propranolol, $150 \mathrm{mg}$ /day of captopril, and $75 \mathrm{mg} /$ day of hydralazine. On admission, her physical examination revealed a

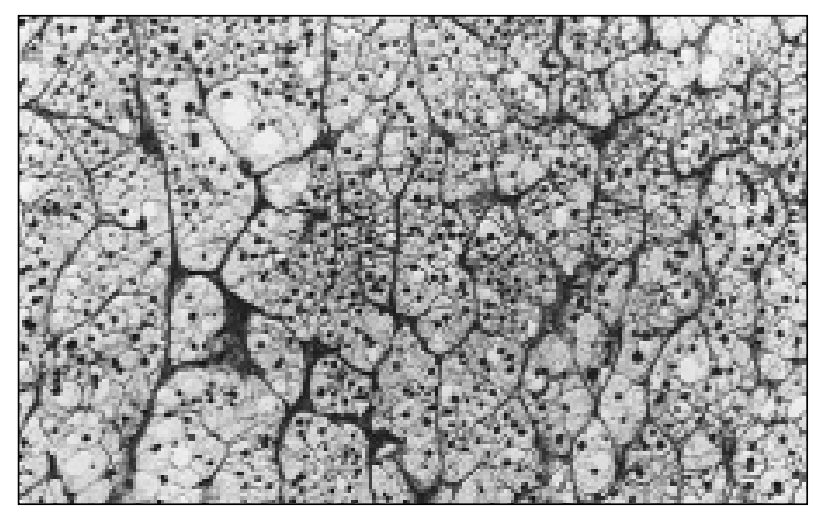

Fig. 1 - Histologic section at the level of the adrenal nodule. The diagnosis of adenoma was established based on the presence of relatively large cells with abundant cytoplasm, scarcity of atypia, and absence of mitoses. Magnification: $10 \mathrm{X}$.

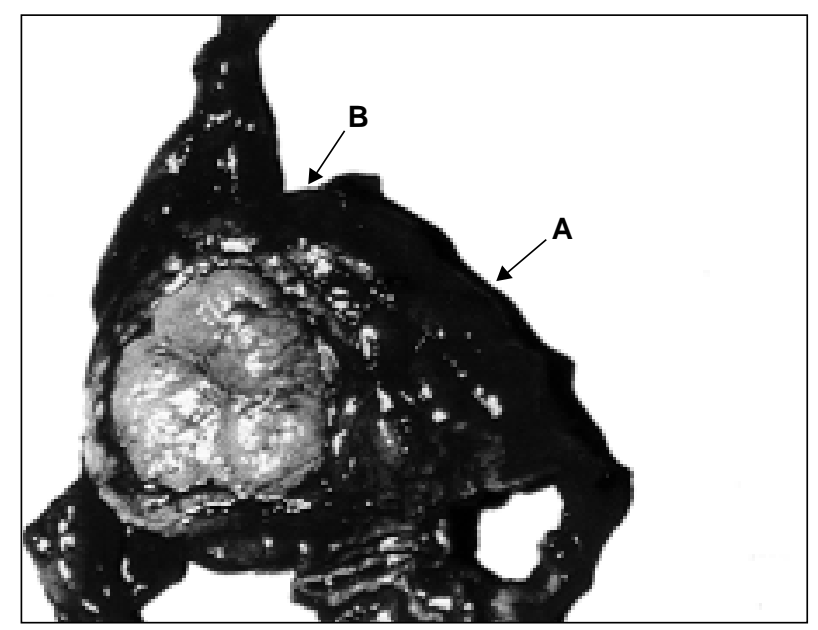

Fig. 2-Histologic section of the adrenal cortex showing an area of normal appearance (A) and the nodule corresponding to the adenoma (B). Magnification: 1.25X. good general condition, heart rate of $70 \mathrm{bpm}$, blood pressure of $240 / 140 \mathrm{mmHg}$, and no other alterations. Funduscopy revealed bilateral papilledema. The electrocardiogram showed signs of hypertrophy of the left chambers. The echocardiogram revealed left ventricular concentric hypertrophy (mass index of $180 \mathrm{~g} / \mathrm{m}^{2}$, posterior wall of $12 \mathrm{~mm}$, diastolic diameter of $50 \mathrm{~mm}$ ) and ejection fraction of 0.71 . The complementary laboratory tests showed normal renal function (serum urea of $25 \mathrm{mg} / \mathrm{dL}$, serum creatinine of $1.1 \mathrm{mg} / \mathrm{dL}$ ) and normal urinary sediment. The abdominal ultrasonography showed kidneys with size and morphology within the normal range. The renal arteriography showed no vascular obstructive lesion. The measurements of vanillylmandelic acid and metanephrines in the 24-hour urine were normal. The initial measurement of plasma electrolytes showed K of $2.3 \mathrm{mEq} / \mathrm{L}$ and $\mathrm{Na}$ of $144 \mathrm{mEq} / \mathrm{L}$. The measurements of the electrolytes in the 24-hour urine (total volume of $920 \mathrm{~mL}$ ) a few days later showed $\mathrm{K}$ of $13 \mathrm{mEq}$ and $\mathrm{Na}$ of $33 \mathrm{mEq}$, concomitantly with plasma K of $3.3 \mathrm{mEq} / \mathrm{mL}$ and plasma $\mathrm{Na}$ of $142 \mathrm{mEq} / \mathrm{L}$. The plasma renin activity was suppressed: $0.25 \mathrm{ng} / \mathrm{mL} / \mathrm{h}$ in the horizontal position and $0.90 \mathrm{ng} / \mathrm{mL} / \mathrm{h}$ after a 2-hour walk (normal value: $5.0 \pm 1.8 \mathrm{ng} / \mathrm{mL} / \mathrm{h}$ ). At the same time, a significant increase in the plasma levels of aldosterone was observed: $56.9 \mathrm{ng} / 100 \mathrm{~mL}$ in the horizontal position (normal range: 1 to $16 \mathrm{ng} / 100 \mathrm{~mL}$ ) and $50.2 \mathrm{ng} /$ $100 \mathrm{~mL}$ after a 2-hour walk (normal range: 4 to $31 \mathrm{ng} / 100 \mathrm{~mL}$ ). Abdominal computerized tomography detected a hypodense nodule of $1.2 \mathrm{~cm}$ of diameter in the right adrenal gland. Blood pressure control during hospitalization was initially unsatisfactory, when the patient maintained high blood pressure levels despite the use of $160 \mathrm{mg} /$ day of propranolol, $150 \mathrm{mg} /$ day of captopril, and $150 \mathrm{mg} /$ day hydralazine. The introduction of $300 \mathrm{mg} /$ day of spironolactone for 10 days did not reduce blood pressure. An appropriate control of blood pressure was only obtained after the combination of minoxidil ( $5 \mathrm{mg}$ every other day), which also significantly improved headache. The patient underwent right adrenalectomy, and a yellow nodule of $1.5 \mathrm{~cm}$ of diameter was found in the cortical zone. The anatomicopathological examination diagnosed adenoma. During the postoperative period, the plasma K levels normalized and the patient was discharged from the hospital asymptomatic. She maintained blood pressure levels of $130 / 90 \mathrm{mmHg}$ taking $50 \mathrm{mg}$ /day of hydrochlorothiazide and $80 \mathrm{mg} /$ day of propranolol. One year later, the patient was readmitted for reassessment. The patient was taking fewer drugs than before the surgery, and her blood pressure remained 140/90 $\mathrm{mmHg}$. Funduscopy revealed no exudates, hemorrhages, or papilledema. A new laboratory assessment revealed plasma potassium levels of $4.6 \mathrm{mEq} / \mathrm{L}$, plasma renin activity at rest of $2 \mathrm{ng} / \mathrm{mL} / \mathrm{h}$, and plasma aldosterone of $10 \mathrm{ng} / 100 \mathrm{~mL}$. Ventricular hypertrophy also decreased (mass index of $110 \mathrm{~g} / \mathrm{m}^{2}$ ).

\section{Discussion}

We report the cases of 2 patients with severe arterial hypertension, 1 with an alteration in funduscopy diagnostic 
of accelerated malignant hypertension (grade 3 in the KeithWagener classification), and another with bilateral papilledema, characteristic of hypertensive encephalopathy, but also present in patients with malignant hypertension, even in the absence of hemorrhages and exudates, which may disappear in a short period of time, as happened with the patient with confirmed accelerated malignant hypertension. Both accelerated malignant hypertension and hypertensive encephalopathy are rare complications of primary aldosteronism, and, therefore, deserve to be discussed. To better discuss the most important aspects of this association from the pathophysiologic point of view, we can consider both patients as having severe arterial hypertension with accelerated or malignant behavior.

Primary aldosteronism due to adrenal adenoma is a rare disease, which affects approximately $1 \%$ of all hypertensive patients ${ }^{3}$. Its prevalence is higher in young females ( 30 to 50 years), and it is usually accompanied by hypokalemia and orthostatic hypotension ${ }^{3}$. Its identification is extremely important, because tumor resection invariably improves blood pressure levels, and, many times completely reverts the hypertensive condition. In addition, accelerated malignant hypertension is an extremely severe condition that causes a great reduction in survival.

The coexistence of primary aldosteronism and accelerated malignant hypertension, although extremely rare, makes the prognosis more favorable, because surgical resection of the tumor eliminates the triggering cause of hypertension. This association should be differentiated from secondary aldosteronism, which is frequently observed in patients with accelerated malignant hypertension due to adrenal hyperactivity ${ }^{2}$. In the initial description by Conn ${ }^{4}$, the association of accelerated malignant hypertension and primary aldosteronism was not observed. Kaplan ${ }^{4}$ reported this association for the first time in 1963.

Since then, several attempts to explain this reduced incidence of malignant transformation of primary aldosteronism have occurred. One of them postulated that renin would be an important factor for the development of the microvascular lesions of malignant hypertension, and renin suppression, in most cases of primary aldosteronism, would prevent malignant transformation ${ }^{2}$. In accelerated malignant hypertension, stimulation of the renin-angiotensin-aldosterone system is known to occur, which may be implicated in the process of malignant transformation ${ }^{5,6}$. In these patients, the initial increase in blood pressure in the afferent glomerular arteriole would lead to a reflex constriction and elevation in the renin levels, followed by an elevation in blood pressure levels, resulting in a vicious cycle with malignant transformation ${ }^{5,6}$. Contrary to this explanation, some authors found elevated plasma renin activity in only $36 \%$ of the patients with accelerated malignant hypertension $^{1}$. Therefore, some researchers have postulated that the rare association of aldosterone-producing adenoma and accelerated malignant hypertension would occur due to the mere infrequent prevalence of each of these diseases separately ${ }^{1}$.
Screening of this secondary form of hypertension is performed through plasma potassium measurement, which is reduced in 70 to $90 \%$ of the patients ${ }^{3}$. The excessive urinary loss of $\mathrm{K}$ is confirmed through potassium level measurement in the 24-hour urine. The patient with accelerated malignant hypertension had an elevated urinary excretion of $\mathrm{K}$ in the presence of reduced plasma potassium levels, while the patient with hypertensive encephalopathy did not have an elevated urinary K level, a fact that may be explained by the low-sodium diet and the relatively small urinary volume $(920 \mathrm{~mL})$.

Laboratory confirmation of primary aldosteronism is achieved through the measurement of plasma renin activity and aldosterone level. Plasma renin activity is suppressed in most cases ${ }^{3}$, although it may be normal or elevated in up to $23 \%$ of the patients ${ }^{2}$. In accelerated malignant hypertension, on the other hand, an increased plasma renin activity is frequently observed ${ }^{2}$. When the 2 diagnoses are associated, the behavior of plasma renin activity is variable.

In this context, according to the proposal by Aloia and Beutow ${ }^{1}$ in 1974, patients with primary aldosteronism and accelerated malignant hypertension may be divided into 3 different groups according to their pathophysiological characteristics. In a first group, the patients would have primary aldosteronism and malignant hypertension with low plasma renin activity. In the second group, primary aldosteronism would lead to benign nephrosclerosis and an increase in the plasma renin activity with consequent malignant transformation. And, in a third group, with the possibility of the so-called tertiary aldosteronism, a continuous stimulation of the adrenal gland by elevated levels of renin would occur, due to the malignant transformation of hypertension, leading to the appearance of a nodule with autonomous production of aldosterone and subsequent renin suppression ${ }^{1}$.

These pathophysiological explanations are difficult to demonstrate, because only a few cases of the association of accelerated malignant hypertension and primary aldosteronism have been reported. However, in these reports, the frequent association of increased plasma renin activity with a worse clinical evolution and a lower response to surgery is worth noting, maybe because of the occurrence of a more advanced degree of nephrosclerosis ${ }^{1}$. Another pertinent observation is the relative lack of reports of malignant hypertension associated with idiopathic hyperplasia, practically all cases being due to adrenal adenoma. In the cases reported, suppression of plasma renin activity and the good response to surgical treatment, which confirmed the diagnosis of adenoma, emphasize these observations.

Therefore, in patients with accelerated malignant hypertension and persistent hypokalemia, the hypothesis of primary aldosteronism due to aldosterone-producing adenoma should be considered. Suppression of plasma renin activity and elevated levels of aldosterone indicate the need to perform imaging examinations, such as computed tomography or magnetic resonance imaging, to elucidate the diagnosis. However, in the presence of malignant 
hypertension, a normal or elevated plasma renin activity does not eliminate the hypothesis of aldosterone-producing adrenal adenoma, and, when this diagnosis is strongly suspected, the investigation should continue, because identification of the condition leads to a specific and highly effective treatment.

\section{References}

1. Aloia JF, Beutow G. Malignant hypertension with aldosterone-producing adenoma. Am J Med Sci 1974; 268: 241-5.

2. Murphy BF, Whitworth JA, Kincaid-Smith P. Malignant hypertension due to an aldosterone-producing adrenal adenoma. Clin Exper Theory Practice 1985; A7: 939-50.

3. Biglieri EG, Irony I, Kater CE. Adrenocortical forms of human hypertension. In Laragh JH, Brenner, BM, eds. Hypertension Pathophysiology, Diagnosis, and Management. New York: Raven Press Ltd., 1990: 1609-23.
4. Kaplan, NM. Primary aldosteronism with malignant hypertension. N Engl J Med 1963; 269: 1282-6.

5. Samani NJ, Ruprai AK, Brammar WJ, Swales JD. The renin gene in patients with malignant hypertension and raised plasma renin activity. Clin Sci 1989; 76: 151-5.

6. Kawazoe N, Eto T, Abe I, et al. Pathophysiology in malignant hypertension: with special reference to the renin-angiotensin system. Clin Cardiol 1987; 10: $513-8$ 